PROCEEDINGS OF THE

AMERICAN MATHEMATICAL SOCIETY

Volume 140, Number 12, December 2012, Pages 4271-4284

S 0002-9939(2012)11291-2

Article electronically published on April 23, 2012

\title{
LAGRANGE INTERPOLATION AT REAL PROJECTIONS OF LEJA SEQUENCES FOR THE UNIT DISK
}

\author{
JEAN-PAUL CALVI AND PHUNG VAN MANH
}

(Communicated by Walter Van Assche)

\begin{abstract}
We show that the Lebesgue constants of the real projection of Leja sequences for the unit disk grow like a polynomial. The main application is the first construction of explicit multivariate interpolation points in $[-1,1]^{N}$ whose Lebesgue constants also grow like a polynomial.
\end{abstract}

\section{INTRODUCTION}

We pursue the work initiated in [4 that aims to construct explicit and (or) easily computable sets of efficient points for multivariate Lagrange interpolation by using the process of intertwining (see below) certain univariate sequences of points. Here, the efficiency of the interpolation points is measured by the growth of their Lebesgue constants (the norms of the interpolation operator). Namely, we look for sets of points $\mathbf{P}_{n} \subset \mathbb{R}^{N}$ - for interpolation by polynomials of total degree at most $n$ - for which the Lebesgue constants $\Delta\left(\mathbf{P}_{n}\right)$ grow at most like a polynomial in $n$. We say that such points are good interpolation points in the sense that if $\Delta\left(\mathbf{P}_{n}\right)=O\left(n^{\alpha}\right)$ with $\alpha \in \mathbb{N}^{\star}$, then, in view of a classical result of Jackson, the Lagrange interpolation polynomials at $\mathbf{P}_{n}$ of any function with $\alpha+1$ continuous (total) derivatives converge uniformly. This is detailed in the paper. In our former work 4], multivariate interpolation points with good Lebesgue constants were constructed on the Cartesian product of many plane compact subsets bounded by sufficiently regular Jordan curves (including, of course, polydiscs) starting from Leja points for the unit disc (see below). Yet, from a practical point of view, especially if we have in mind applications to numerical analysis, the real case is more interesting. It is the purpose of this paper to exhibit explicit interpolation points in $[-1,1]^{N}$ with Lebesgue constants growing at most like a polynomial. As far as we know, this is the first general construction of such points. This will be done by suitably modifying the methods employed in [4. Actually, the unidimensional points will be taken as the projections on the real axis of the points of a Leja sequence. We shall first show how to describe (and compute) these points and, in particular, prove that they are Chebyshev-Lobatto points (of increasing degree) arranged in a certain manner. We shall then study their Lebesgue constants to prove that they grow at most like $n^{3} \log n$, where $n$ is the degree of interpolation. The passage to the multivariate case is identical to that shown in 4 and will not be detailed.

Received by the editors February 21, 2011 and, in revised form, June 3, 2011.

2010 Mathematics Subject Classification. Primary 41A05, 41A63.

Key words and phrases. Lagrange interpolation, Lebesgue constants, Leja sequences.

(C)2012 American Mathematical Society

Reverts to public domain 28 years from publication 
Notation. We refer to [4 for basic definitions on Lagrange interpolation theory. Let us just indicate that, given a finite set $A$, we write $w_{A}:=\prod_{a \in A}(\cdot-a)$. The fundamental Lagrange interpolation polynomial (FLIP) for $a \in A$ is denoted by $\ell(A, a ; \cdot)$. We have

$$
\ell(A, a ; \cdot)=\prod_{b \in A, b \neq a}(\cdot-b) /(a-b)=w_{A} /\left(w_{A}^{\prime}(a)(\cdot-a)\right) .
$$

The Lagrange interpolation polynomial of $f$ is $\mathbf{L}[A ; f]=\sum_{a \in A} f(a) \ell(A, a ; \cdot)$ and the norm of $\mathbf{L}[A ; \cdot]$ as an operator on $C(K)$ (where $K$ is a compact subset containing $A)$ is the Lebesgue constant $\Delta(A)=\Delta(A, K)$. It is known that $\Delta(A)=$ $\max _{z \in K} \sum_{a \in A}|\ell(A, a ; z)|$.

We shall denote by $M, M^{\prime}$, etc., constants independent of the relevant parameters. Occurrences of the same letter in different places do not necessarily refer to the same constant.

\section{LeJa Sequences AND their PROJeCtions on the REAL AXis}

2.1. Leja sequences for the unit disk. We briefly recall the definition and the structure of a Leja sequence for the unit disk $D=\{|z| \leq 1\} \subset \mathbb{C}$. A $k$-tuple $E_{k}=\left(e_{0}, \ldots, e_{k-1}\right) \in D^{k}$ with $e_{0}=1$ is a $k$-Leja section for $D$ if, for $j=1, \ldots, k-1$, the $(j+1)$-st entry $e_{j}$ maximizes the product of the distances to the $j$ previous points, that is,

$$
\prod_{m=0}^{j-1}\left|e_{j}-e_{m}\right|=\max _{z \in D} \prod_{m=0}^{j-1}\left|z-e_{m}\right|, \quad j=1, \ldots, k-1 .
$$

The maximum principle implies that the points $e_{i}$ actually lie on the unit circle $\partial D$. A sequence $E=\left(e_{k}: k \in \mathbb{N}\right)$ for which $E_{k}:=\left(e_{0}, \ldots, e_{k-1}\right)$ is a $k$-Leja section for every $k \in \mathbb{N}^{\star}$ is called a Leja sequence (for $D$ ). Of course, the points of a Leja sequence are pairwise distinct.

The structure of a Leja sequence is studied in [1, where one can find the following result.

Theorem 2.1 (Białas-Cież and Calvi). A Leja sequence is characterized by the following two properties:

(1) The underlying set of a $2^{n}$-Leja section for $D$ is formed of the $2^{n}$-th roots of unity.

(2) If $E_{2^{n+1}}$ is a $2^{n+1}$-Leja section, then there exist a $2^{n}$-root $\rho$ of -1 and a $2^{n}$-Leja section $E_{2^{n}}^{(1)}$ such that $E_{2^{n+1}}=\left(E_{2^{n}}, \rho E_{2^{n}}^{(1)}\right)$. is

Here, the second assertion means the following. The element of index $s$ in $E_{2^{n+1}}$

(1) the element of index $s$ in $E_{2^{n}}$ when $0 \leq s \leq 2^{n}-1$,

(2) $\rho$ times the element of index $\left(s-2^{n}\right)$ in $E_{2^{n}}^{(1)}$ when $2^{n} \leq s \leq 2^{n+1}-1$.

Repeated applications of Theorem 2.1 show that if $d=2^{n_{0}}+2^{n_{1}}+\cdots+2^{n_{r}}$ with $n_{0}>n_{1}>\cdots>n_{r} \geq 0$, then

$$
\begin{aligned}
E_{d} & =\left(E_{2^{n_{0}}}, \rho_{0} E_{d-2^{n_{0}}}^{(1)}\right)=\left(E_{2^{n_{0}}}, \rho_{0} E_{2^{n_{1}}}^{(1)}, \rho_{1} \rho_{0} E_{d-2^{n_{0}}-2^{n_{1}}}^{(2)}\right) \\
& =\cdots=\left(E_{2^{n_{0}}}, \rho_{0} E_{2^{n_{1}}}^{(1)}, \rho_{1} \rho_{0} E_{2^{n_{2}}}^{(2)}, \ldots, \rho_{r-1} \cdots \rho_{1} \rho_{0} E_{2^{n_{r}}}^{(r)}\right),
\end{aligned}
$$


where each $E_{2^{n_{j}}}^{(j)}$ consists of a complete set of $2^{n_{j}}$-roots of unity, arranged in a certain order (actually, a $2^{n_{j}}$-Leja section), and $\rho_{j}$ is a $2^{n_{j}}$-th root of -1 .

2.2. Projections of Leja sequences. We are interested in polynomial interpolation at the projections on the real axis of the points of a Leja sequence. We eliminate repeated values and this somewhat complicates the description of the resulting sequence. We use $\Re(\cdot)$ to denote the real part of a complex number (or sequence).

Definition 2.2. A sequence $X$ (in $[-1,1]$ ) is said to be an $\Re$-Leja sequence if there exists a Leja sequence $E=\left(e_{k}: k \in \mathbb{N}\right)$ such that $X$ is obtained by eliminating repetitions in $\Re\left(e_{k}: k \in \mathbb{N}\right)$. Here, we mean that the entry $\Re\left(e_{j}\right)$ is eliminated whenever there exists $i<j$ such that $\Re\left(e_{j}\right)=\Re\left(e_{i}\right)$. We write $X=X(E)$.

In particular $X(E)$ is a subsequence of $\Re\left(e_{k}: k \in \mathbb{N}\right)$. Since for every $n \in \mathbb{N}$, the underlying set of a $2^{n+1}$-Leja section is a complete set of $2^{n+1}$-st roots of unity (Theorem 2.1), the corresponding real parts form the set $\mathcal{L}_{2^{n}}$ of Chebyshev-Lobatto (or Gauss-Lobatto) points of degree $2^{n}$,

$$
\mathcal{L}_{2^{n}}=\left\{\cos \left(j \pi / 2^{n}\right): j=0, \ldots, 2^{n}\right\} .
$$

These points are the extremal points of the usual Chebyshev polynomial (of degree $2^{n}$ ) and are sometimes referred to as the "Chebyshev extremal points".

For future reference, we state this observation as a lemma.

Lemma 2.3. Let $X$ be an $\Re$-Leja sequence. For every $n \in \mathbb{N}$, the underlying set of $X_{2^{n}+1}=\left(x_{0}, \ldots, x_{2^{n}}\right)$ is the set of Chebyshev-Lobatto points $\mathcal{L}_{2^{n}}$.

Theorem 2.4 below gives two descriptions of $\Re$-Leja sequences. The first one is particularly adapted to the computations of $\Re$-Leja sequences when one is given Leja sequences. Examples of easily computable (and explicit) Leja sequences can be found in [4, Lemma 2]. In Figure1(I), we show the first 16 points of a Leja sequence $E$ and the first 9 points of the corresponding $\Re$-Leja sequence $X(E)$. (The Leja sequence we use is given by the rule $E_{2}=(1,-1)$ and $E_{2^{n+1}}=\left(E_{2^{n}}, \exp \left(i \pi / 2^{n}\right) E_{2^{n}}\right)$.)

The concatenation of tuples is denoted by $\wedge$,

$$
\left(x_{1}, \ldots, x_{m}\right) \wedge\left(y_{1}, \ldots, y_{n}\right):=\left(x_{1}, \ldots, x_{m}, y_{1}, \ldots, y_{n}\right) .
$$

For every sequence of complex numbers $S=\left(s_{k}: k \in \mathbb{N}\right)$ we define $S(j: k):=$ $\left(s_{j}, s_{j+1}, \ldots, s_{k}\right)$. As before, $S_{k}:=S(0: k-1)$.

Theorem 2.4. A sequence $X=\left(x_{k}: k \in \mathbb{N}\right)$ is an $\Re$-Leja sequence if and only if there exists a Leja sequence $E=\left(e_{k}: k \in \mathbb{N}\right)$ such that

$$
X=(1,-1) \wedge \bigwedge_{j=1}^{\infty} \Re\left(E\left(2^{j}: 2^{j}+2^{j-1}-1\right)\right) .
$$

Equivalently, $x_{k}=\Re\left(e_{\phi(k)}\right), k \in \mathbb{N}$, with $\phi(0)=0, \phi(1)=1$ and

$$
\phi(k)=\left\{\begin{array}{ll}
\frac{3 k}{2}-1 & k=2^{n} \\
2^{\left\lfloor\log _{2}(k)\right\rfloor}+k-1 & k \neq 2^{n}
\end{array}, \quad k \geq 2,\right.
$$

where $\lfloor\cdot\rfloor$ is used for the ordinary floor function. 
Proof. Let $X=X(E)$ with $E=\left(e_{s}: s \in \mathbb{N}\right)$. We prove that if $2^{j} \leq k<2^{j}+2^{j-1}$, then $\Re\left(e_{k}\right)$ does not appear in $\Re\left(E_{k}\right)$ and therefore provides a new point for $X$. To do that, since $e_{k}$ itself does not belong to $E_{k}$, it suffices to check that $\overline{e_{k}}$ is not a point of $E_{k}$, equivalently $e_{k} \neq \overline{e_{s}}, 0 \leq s \leq k-1$. If $s<2^{j}$, then $\overline{e_{s}}$ is a $2^{j}$-th root of unity whereas $e_{k}$ is not. On the other hand, if $2^{j} \leq s \leq k-1$, then, in view of Theorem 2.1, we have

$$
E_{2^{j+1}}=\left(E_{2^{j}}, \rho E_{2^{j}}^{(1)}\right)=\left(E_{2^{j}}, \rho E_{2^{j-1}}^{(1)}, \rho \rho^{\prime} E_{2^{j-1}}^{(2)}\right) ;
$$

hence $e_{k}$ and $e_{s}$ appear in the second tuple so that $e_{k}=\rho a$ and $e_{s}=\rho b$, where $\rho$ is a $2^{j}$-th root of -1 and both $a$ and $b$ are $2^{j-1}$-st roots of unity. The relation $e_{k}=\overline{e_{s}}$ yields $\rho / \bar{\rho}=\bar{b} / a$. The argument of the first number is of the form $2(2 l+1) \pi / 2^{j}$ and the argument of the second one is $2 t \pi / 2^{j-1}$ (with $l, t \in \mathbb{Z}$ ). Equality is therefore impossible.

Now, in view of Lemma 2.3 from $E_{2^{j+1}}$ we obtain $2^{j}+1$ points for $X$, namely the points in $\mathcal{L}_{2^{j}}$ arranged in a certain way. Yet, the $2^{j}+1$ first points of $X$ are already given by $E_{2^{j}}\left(2^{j-1}+1\right.$ points $)$ together with, according to the first part of this proof, the $2^{j-1}$ points $\Re\left(e_{k}\right), 2^{j} \leq k<2^{j}+2^{j-1}$. This implies that if $2^{j}+2^{j-1} \leq k<2^{j+1}$, then $\Re\left(e_{k}\right)$ is not a new point for $X$. This achieves the proof of (2.3).

To prove (2.4) we observe that, in view of (2.3), we have

$$
\Re\left(e_{2^{k}+i}\right)=x_{2^{k-1}+i+1}, \quad 0 \leq i \leq 2^{k-1}-1 .
$$

Hence $\phi\left(2^{k-1}+i+1\right)=2^{k}+i$ and the expression for $\phi$ easily follows.

Corollary 2.5 (to the proof). If $X=X(E)$, then $X\left(2^{n}+1: 2^{n+1}\right)=\Re\left(E\left(2^{n+1}\right.\right.$ : $\left.\left.2^{n+1}+2^{n}-1\right)\right)$.

Decompositions (2.2) and (2.3) are fundamental to this work. In particular, the binary expansion of $k$ will be used in the study of the tuple $X(0: k)$.

Note that of course the decomposition would be different if we projected the Leja points on another segment, say on $\left[-e^{i \theta}, e^{i \theta}\right]$. The distribution of the projected points in general depends on arithmetic properties of $\theta$. We shall not discuss the general case in this paper.

Finally, let us point out that our $\Re$-Leja sequences are not Leja sequences for the interval. It can easily be shown that they are pseudo Leja sequences. A pseudo Leja sequence for a plane compact set $K$ is a sequence $\left(a_{n}\right)$ in $K$ that satisfies an inequality of the form

$$
\max _{z \in K} \prod_{i=0}^{d}\left|z-a_{i}\right| \leq M_{d+1} \prod_{i=0}^{d}\left|a_{d+1}-a_{i}\right|, \quad d \geq 0,
$$

where $M_{d}$ is a sequence of subexponential growth $\left(M_{d}^{1 / d} \rightarrow 1\right.$ as $\left.d \rightarrow \infty\right)$. We refer to 1 for results on pseudo Leja sequences. There is no known expression for Leja points for the interval. For that matter, such an expression is very unlikely to exist. Note however that by a result of Taylor and Totik 9], the Lebesgue constants of a Leja sequence for the interval grow at most subexponentially. A referee also pointed out that, in a recent work, A. Goncharov [6] arranged ordinary Chebyshev points for $[-1,1]$ into a certain sequence and showed that the Lebesgue constants for this sequence grow subexponentially. They are actually shown to be $O\left(k^{\log k / \log 3} k^{8}\right)$ as the degree $k$ tends to $\infty$. Goncharov also shows that the Lebesgue constants 
for this set of points cannot grow polynomially. These sequences could be used to construct further multivariate interpolation points with Lebesgue constants that grow subexponentially. This is not sufficient to derive approximation results for differentiable functions yet implies optimal approximation properties for analytic functions; see [1, Subsection 2.3].

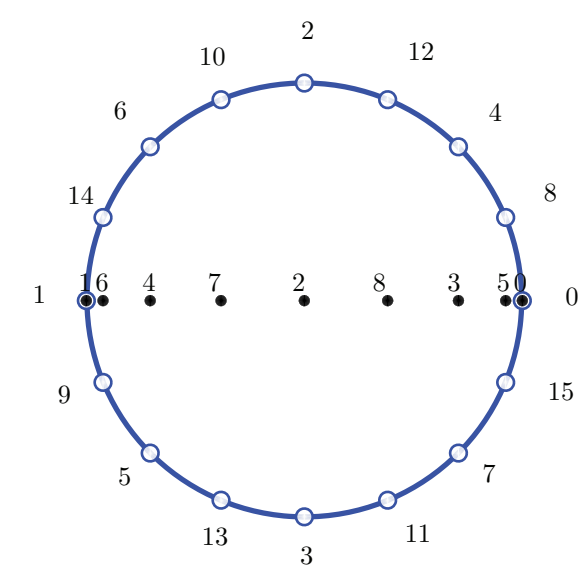

(I) First 9 points of an $\Re$-Leja sequence.

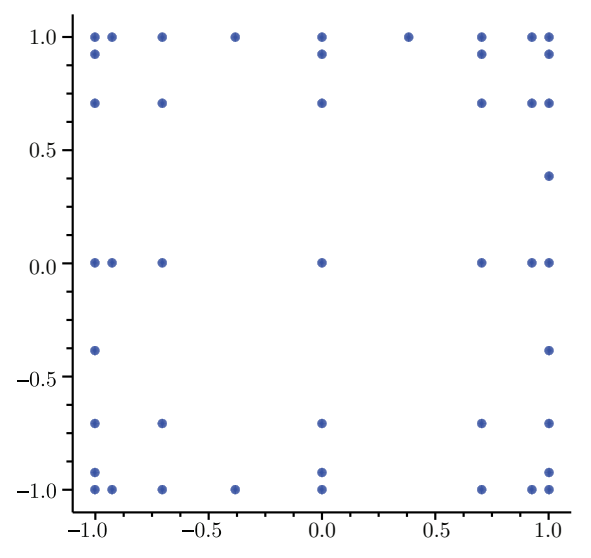

(II) 45 interpolation points obtained as the intertwining of the points in (I) with themselves.

FigurE 1. Examples of points from an $\Re$-Leja sequence and their intertwining.

\section{Lebesgue constants of $\Re$-LeJA SEQUences}

3.1. The upper bound and its consequences. Recall that given a set $A$ of $n+1$ interpolation points in $[-1,1]$ and $f \in \mathrm{C}^{s}([-1,1])$, the Lebesgue inequality, together with the Jackson theorem [8, Theorem 1.5], yields the well-known estimate

$$
\max _{[-1,1]}\|f-\mathbf{L}[A ; f]\| \leq M(1+\Delta(A)) \omega\left(f^{(s)}, 1 / n\right) / n^{s},
$$

where $\omega\left(f^{(s)}, \cdot\right)$ denotes the modulus of continuity of $f^{(s)}$ and $M$ does not depend either on $A$ or $n$. The following theorem implies in particular that interpolation polynomials at the points of any $\Re$-Leja sequence converge uniformly on $[-1,1]$ to the interpolated function as soon as it belongs to $\mathrm{C}^{4}([-1,1])$. A weaker consequence is that the discrete measure $\mu_{d}:=\frac{1}{d+1} \sum_{i=0}^{d}\left[x_{i}\right]$ associated to the $\Re$-Leja sequence $X=\left(x_{s}: s \in \mathbb{N}\right)$ weakly converges to the 'arcsin' distribution on $[-1,1]$ which is the equilibrium measure of the interval. Here $\left[x_{i}\right]$ denotes the Dirac measure at $x_{i}$.

Theorem 3.1. Let $X$ be an $\Re$-Leja sequence. The Lebesgue constant $\Delta\left(X_{k}\right)$ for the interpolation points $x_{0}, \ldots, x_{k-1}$ satisfies the following estimate:

$$
\Delta\left(X_{k}\right)=O\left(k^{3} \log k\right), \quad k \rightarrow \infty .
$$


The construction of good multivariate interpolation points is derived as follows. We start from $N \Re$-Leja sequences $X^{(j)}=\left(x_{k}^{(j)}: k \in \mathbb{N}\right), j=1, \ldots, N$. These $N$ sequences need not be distinct. We define $\mathbf{P}_{k} \subset[-1,1]^{N}$ as

$$
\mathbf{P}_{k}=\left\{x_{\alpha}=\left(x_{\alpha_{1}}^{(1)}, \ldots, x_{\alpha_{N}}^{(N)}\right): \sum_{j=1}^{N} \alpha_{j} \leq k\right\} .
$$

It is known 3] that this is a valid set for interpolation by $N$-variable polynomials of degree at most $k$. Actually, $\mathbf{P}_{k}$ is the underlying set of the intertwining of the univariate tuples $X^{(j)}(0: k), j=1, \ldots, N$. We refer to [4] for details on this definition and to [3] for a general discussion of the intertwining process. Let us just emphasize that, in order to provide good points, the method requires us to use sequences of interpolation points (we add one point when we go from degree $k$ to $k+1$ ) rather than arrays (the classical case: all the points change when we change the degree). The reason for this is explained in [4].

In Figure 1 (II), we show the points of a set $\mathbf{P}_{8}$ constructed with the first 9 points of the $\Re$-Leja sequence in (I).

Theorem 3.2. The Lebesgue constants $\Delta\left(\mathbf{P}_{k}\right)$ grow at most like a polynomial in $k$ as $k \rightarrow \infty$.

Proof. The proof of [4, Theorem 16] works as well in this case.

Just as in the univariate case, the multivariate versions of the Lebesgue inequality and the Jackson theorem, together with Theorem 3.2 imply that Lagrange interpolants of sufficiently smooth functions converge uniformly. Examining the terms in the proof of [4, Theorem 16], we find

$$
\Delta\left(\mathbf{P}_{k}\right)=O\left(k^{\left(N^{2}+11 N-6\right) / 2} \log ^{N} k\right), \quad k \rightarrow \infty,
$$

which gives a more precise idea of the required level of smoothness. This bound however is certainly pessimistic.

In the rest of the paper, unless otherwise stated, the Lebesgue constants are computed with respect to the interval $[-1,1]$.

3.2. Outline of the proof of Theorem 3.1. We take advantage of the structure of the points of an $\Re$-Leja sequence. The first step is a simple algebraic observation. We use the notation recalled at the end of the introduction.

Lemma 3.3. Let $N=N_{0} \cup \cdots \cup N_{s-1}$, where the $N_{i}$ form a partition of the finite set $N \subset \mathbb{R}$. We have

$$
\ell(N, a ; \cdot)=\frac{w_{N \backslash N_{i}}}{w_{N \backslash N_{i}}(a)} \ell\left(N_{i}, a ; \cdot\right), \quad a \in N_{i}, \quad i=0, \ldots, s-1 .
$$

Consequently,

$$
\Delta(N) \leq \sum_{i=0}^{s-1} \max _{x \in K, a \in N_{i}}\left|\frac{w_{N \backslash N_{i}}(x)}{w_{N \backslash N_{i}}(a)}\right| \Delta\left(N_{i}\right),
$$

where the Lebesgue constants are computed with respect to a compact set $K$ containing $N$.

Proof. We readily check that the polynomial on the right-hand side of (3.1) satisfies the defining properties of $\ell(N, a ; \cdot)$. The estimate for the Lebesgue constant $\Delta(N)$ follows from the definition together with the formula for the FLIPs. 
Given an $\Re$-Leja sequence $X=X(E)$, to estimate $\Delta\left(X_{k}\right), 2^{n}+1<k \leq 2^{n+1}$, we shall first apply the lemma with a partition of $X_{k}$ into two subsets, namely $A=X\left(0: 2^{n}\right)=\mathcal{L}_{2^{n}}$ and $B=X\left(2^{n}+1: k-1\right)$. Here and below, when there is no risk of misunderstanding, we confuse a tuple with its underlying set. In other words, when we write $T=Y$ with $T$ a tuple and $Y$ a set, we mean that $Y$ is formed of the entries of $T$. Our choice for $A$ and $B$, of course, is motivated by the fact that the Chebyshev-Lobatto points are excellent interpolation points for which there is a large amount of information available; see below. With this choice, Lemma 3.3 gives

$$
\Delta\left(X_{k}\right) \leq \Delta(A) \max _{x \in[-1,1], a \in A}\left|\frac{w_{B}(x)}{w_{B}(a)}\right|+\Delta(B) \max _{x \in[-1,1], b \in B}\left|\frac{w_{A}(x)}{w_{A}(b)}\right| .
$$

The factors depending on the first subset $A$ in (3.3) will be easily estimated. The more difficult part will be to estimate $w_{B}$ and $\Delta(B)$. To do that, we shall use a partition of $B$ and still have recourse to Lemma 3.3 in its most general form. We point out however that our method is unlikely, it seems, to give the best estimates. Intuitively, we think that sharp estimates cannot be obtained by separating the interpolation points into two or more groups.

It is not difficult to see that the Lebesgue constants $\Delta\left(X_{k}\right)$ cannot grow slower than $k$. This is explained in subsection 3.4 .

3.3. Interpolation at Chebyshev-Lobatto points. We collect a few results on Chebyshev-Lobatto points. First, since they are the extremal points of the Chebyshev polynomials, we have

$$
w_{\mathcal{L}_{d}}(x)=\left(x^{2}-1\right) d^{-1} T_{d}^{\prime}(x), \quad x \in \mathbb{R},
$$

where $T_{d}$ denotes the monic Chebyshev polynomial of degree $d$. From $2^{d-1} T_{d}(\cos \theta)$ $=\cos d \theta$ we readily find

$$
w_{\mathcal{L}_{d}}(\cos \theta)=-2^{1-d} \sin \theta \sin d \theta, \quad \theta \in \mathbb{R} .
$$

A classical result of Ehlich and Zeller [5, 2] ensures that

$$
\Delta\left(\mathcal{L}_{d}\right)=O(\log d), \quad d \rightarrow \infty .
$$

Lemma 3.4. Let $X=X(E)$ be an $\Re$-Leja sequence. If $2^{n}+1<k \leq 2^{n+1}$, $A=X\left(0: 2^{n}\right), B=X\left(2^{n}+1: k-1\right)$ and $K=[-1,1]$ then

$$
\max _{x \in K, b \in B} \frac{\left|w_{A}(x)\right|}{\left|w_{A}(b)\right|} \leq 1 / \sin \left(\pi / 2^{n+1}\right) .
$$

Proof. If $x=\cos t$ and $b=\cos \beta$, then, since $A=\mathcal{L}_{2^{n}}$, in view of (3.4), we have

$$
\left|w_{A}(x) / w_{A}(b)\right|=\left|\sin t \sin \left(2^{n} t\right)\right| /\left|\sin \beta \sin \left(2^{n} \beta\right)\right| \leq 1 /\left|\sin \beta \sin \left(2^{n} \beta\right)\right| .
$$

Hence, it suffices to check that

$$
\left|\sin \beta \sin \left(2^{n} \beta\right)\right| \geq \sin \left(\pi / 2^{n+1}\right) .
$$

Since $b=x_{j}$ with $2^{n}+1 \leq j \leq k-1<2^{n+1}$, equation (2.4) gives

$$
x_{j}=\Re\left(e_{2^{n}+j-1}\right)=\Re\left(e_{2^{n+1}+u-1}\right), \quad u=j-2^{n}, 1 \leq u \leq 2^{n}-1 .
$$

Theorem 2.1 says that $e_{2^{n+1}+u-1}=\rho z$, where $\rho$ is a $2^{n+1}$-st root of -1 and $z$ is a $2^{n}$-th root of 1 . This means that the angle $\beta$ that gives $b=x_{j}$ is of the form

$$
\beta=(2 \tau+1) \pi / 2^{n+1}+2 \tau^{\prime} \pi / 2^{n} \quad \text { with } \tau, \tau^{\prime} \in \mathbb{Z}
$$


It follows that $\left|\sin 2^{n} \beta\right|=1$ and $|\sin \beta| \geq \sin \left(\pi / 2^{n+1}\right)$. This gives inequality (3.6) and concludes the proof of the lemma.

3.4. A lower bound. We now show that when we remove one point from $\mathcal{L}_{d}$, the Lebesgue constants grow significantly faster. Write $a_{i}=\cos (i \pi / d)$. Suppose that $A_{j}:=\mathcal{L}_{d} \backslash\left\{a_{j}\right\}$ with $1 \leq j \leq d-1$ (so that we remove a point different from 1 and $-1)$. We compute the values of the FLIPs for $A_{j}$ at the missing point $a_{j}$. From $w_{\mathcal{L}_{d}}(\cdot)=w_{A_{j}}(\cdot)\left(\cdot-a_{j}\right)$ we get

$$
w_{A_{j}}\left(a_{j}\right)=w_{\mathcal{L}_{d}}^{\prime}\left(a_{j}\right) \text { and } w_{\mathcal{L}_{d}}^{\prime}\left(a_{i}\right)=w_{A_{j}}^{\prime}\left(a_{i}\right)\left(a_{i}-a_{j}\right), \quad i \neq j .
$$

Hence,

$$
\ell\left(A_{j}, a_{i} ; a_{j}\right)=\frac{w_{A_{j}}\left(a_{j}\right)}{\left(a_{j}-a_{i}\right) w_{A_{j}}^{\prime}\left(a_{i}\right)}=-\frac{w_{\mathcal{L}_{d}}^{\prime}\left(a_{j}\right)}{w_{\mathcal{L}_{d}}^{\prime}\left(a_{i}\right)} .
$$

Yet, as easily follows from (3.4),

$$
w_{\mathcal{L}_{d}}^{\prime}\left(a_{i}\right)= \pm 2^{1-d} d, \quad i=1, \ldots, d-1 .
$$

Hence $\left|\ell\left(A_{j}, a_{i} ; a_{j}\right)\right|=1$ for $d-2$ values of $i$, namely for $i=1, \ldots, d-1, i \neq j$. Consequently,

$$
\Delta\left(A_{j}\right) \geq \sum_{i=1, i \neq j}^{d-1}\left|\ell\left(A_{j}, a_{i} ; a_{j}\right)\right|=d-2 .
$$

Here is the consequence about our $\Re$-Leja sequences. If $X$ is an $\Re$-Leja sequence, then $X\left(0: 2^{n}-1\right)$ is formed of all the Chebyshev-Lobatto points of degree $2^{n}$ with only one missing, and this missing point is different from 1 and -1 (as soon as $n \geq 2$ ), which are the first two points of the sequence. Hence, according to (3.7), $\Delta\left(X\left(0: 2^{n}-1\right)\right) \geq 2^{n}-2$, which shows that the Lebesgue constants $\Delta\left(X_{k}\right)$ cannot grow slower than $k$.

3.5. Interpolation at modified Chebyshev points. We introduce other sets of interpolation points that will naturally come into play when dealing with $B=$ $X\left(2^{n}+1: k-1\right)$. When $\cos \beta$ is not an extremal point of $T_{d}$, that is, $\cos \beta \notin \mathcal{L}_{d}$, then the equation $T_{d}(x)=T_{d}(\cos \beta)$ has $d$ roots in $[-1,1]$. The set of these roots - which we call modified Chebyshev points - will be denoted by $\mathcal{T}_{d}^{(\beta)}$. Since the change of variable $x=\cos t$ transforms the equation to $\cos d t=\cos d \beta$, we have

$$
\mathcal{T}_{d}^{(\beta)}=\left\{\cos \beta_{j}: \beta_{j}:=\beta+2 j \pi / d, j=0, \ldots, d-1\right\} .
$$

The following result is probably known, but we are unable to provide references.

Lemma 3.5. We have $\Delta\left(\mathcal{T}_{d}^{(\beta)}\right)=O(\log d /|\sin d \beta|)$ as $d \rightarrow \infty$, and the constant involved in $O$ does not depend on $\beta$. Equivalently, there exists $M$ such that

$$
\Delta\left(\mathcal{T}_{d}^{(\beta)}\right) \leq M \log (d+1) /|\sin d \beta|, \quad d \geq 1 .
$$

Proof. First, since

$$
w_{\mathcal{T}_{d}^{(\beta)}}(x)=T_{d}(x)-T_{d}(\cos \beta)
$$

(see the introduction for the notation $w_{\mathcal{T}_{d}^{(\beta)}}$ ), we have

$$
\ell\left(\mathcal{T}_{d}^{(\beta)}, \cos \beta_{j} ; x\right)=\frac{T_{d}(x)-T_{d}(\cos \beta)}{T_{d}^{\prime}\left(\cos \beta_{j}\right)\left(x-\cos \beta_{j}\right)}=\frac{\sin \beta_{j}(\cos d t-\cos d \beta)}{d \sin d \beta\left(\cos t-\cos \beta_{j}\right)}, \quad x=\cos t .
$$


A use of the sum-to-product identity for cosines now yields

$$
\ell\left(\mathcal{T}_{d}^{(\beta)}, \cos \beta_{j} ; x\right)=\frac{\sin \beta_{j}}{d \sin d \beta} \frac{\sin (d(t+\beta) / 2) \sin (d(t-\beta) / 2)}{\sin \left(\left(t+\beta_{j}\right) / 2\right) \sin \left(\left(t-\beta_{j}\right) / 2\right)} .
$$

Yet, we also have

$$
\sin \beta_{j}=\sin \left(\left(t+\beta_{j}\right) / 2\right) \cos \left(\left(t-\beta_{j}\right) / 2\right)-\sin \left(\left(t-\beta_{j}\right) / 2\right) \cos \left(\left(t+\beta_{j}\right) / 2\right) .
$$

Using this in (3.8), we obtain after simplification,

$$
\begin{aligned}
\ell\left(\mathcal{T}_{d}^{(\beta)}, \cos \beta_{j} ; x\right)=\frac{1}{d \sin d \beta} & \left\{\frac{\cos \left(\left(t-\beta_{j}\right) / 2\right) \sin (d(t+\beta) / 2) \sin (d(t-\beta) / 2)}{\sin \left(\left(t-\beta_{j}\right) / 2\right)}\right. \\
& \left.-\frac{\cos \left(\left(t+\beta_{j}\right) / 2\right) \sin (d(t+\beta) / 2) \sin (d(t-\beta) / 2)}{\sin \left(\left(t+\beta_{j}\right) / 2\right)}\right\} .
\end{aligned}
$$

It follows that

$$
\left|\ell\left(\mathcal{T}_{d}^{(\beta)}, \cos \beta_{j} ; x\right)\right| \leq \frac{1}{d|\sin d \beta|}\left\{\left|\frac{\sin (d(t-\beta) / 2)}{\sin \left(\left(t-\beta_{j}\right) / 2\right)}\right|+\left|\frac{\sin (d(t+\beta) / 2)}{\sin \left(\left(t+\beta_{j}\right) / 2\right)}\right|\right\}, \quad x=\cos t .
$$

Hence,

$$
\Delta\left(\mathcal{T}_{d}^{(\beta)}\right) \leq \frac{1}{|\sin d \beta|}\left\{\max _{t \in \mathbb{R}} F(t-\beta)+\max _{t \in \mathbb{R}} F(t+\beta)\right\}=\frac{2}{|\sin d \beta|} \max _{t \in \mathbb{R}} F(t),
$$

where

$$
F(t)=\frac{1}{d} \sum_{j=0}^{d-1}\left|\frac{\sin (d t / 2)}{\sin ((t-2 j \pi / d) / 2)}\right| .
$$

But $\max _{t \in \mathbb{R}} F(t)$ is exactly the Lebesgue constant for the $d$-th roots of unity (on the unit circle) which is known to be $O(\log d)$; see [7].

\section{Proof of Theorem 3.1}

4.1. Further reduction. We use Lemma 3.4 and the classical estimate 3.5 of Ehlich and Zeller in (3.3) to obtain the following lemma.

Lemma 4.1. Let $X$ be an $\Re$-Leja sequence and let $2^{n}+1<k \leq 2^{n+1}$. If $A=$ $X\left(0: 2^{n}\right)$ and $B=X\left(2^{n}+1: k-1\right)$, then

$$
\Delta\left(X_{k}\right) \leq M \log 2^{n} \max _{x \in[-1,1], a \in A} \frac{\left|w_{B}(x)\right|}{\left|w_{B}(a)\right|}+\frac{\Delta(B)}{\sin \left(\pi / 2^{n+1}\right)},
$$

where $M$ does not depend on $k$.

The remaining required information is collected in the following two theorems.

Theorem 4.2. Let $X$ be an $\Re$-Leja sequence and let $2^{n}+1<k \leq 2^{n+1}$. If $A=X\left(0: 2^{n}\right)$ and $B=X\left(2^{n}+1: k-1\right)$, then

$$
\max _{x \in[-1,1], a \in A} \frac{\left|w_{B}(x)\right|}{\left|w_{B}(a)\right|} \leq 2^{2 n+2} .
$$

Theorem 4.3. Let $X$ be an $\Re$-Leja sequence and let $2^{n}+1<k \leq 2^{n+1}$. If $B=X\left(2^{n}+1: k-1\right)$, then

$$
\Delta(B) \leq M^{\prime} 2^{2 n} \log 2^{n},
$$

where the constant $M^{\prime}$ does not depend on $k$. 
End of proof of Theorem 3.1. When $k-1$ is a power of 2, the points of $X_{k}$ form a complete set of Chebyshev-Lobatto points, and the bound is implied by Ehlich and Zeller's estimate (3.5). We assume $2^{n}+1<k \leq 2^{n+1}$. Using Theorems 4.2 and 4.3 in (4.1), we obtain

$$
\Delta\left(X_{k}\right) \leq M 2^{2 n+2} \log 2^{n}+M^{\prime} \frac{2^{2 n} \log 2^{n}}{\sin \left(\pi / 2^{n+1}\right)} .
$$

Since $n=\left\lfloor\log _{2}(k)\right\rfloor$ (or $\left\lfloor\log _{2}(k)\right\rfloor-1$ in the case $\left.k=2^{n+1}\right)$ and $1 / \sin \left(\pi / 2^{n+1}\right)=$ $O\left(2^{n}\right)$, this readily gives the existence of a constant $M^{\prime \prime}$ (independent of $k$ ) such that

$$
\Delta\left(X_{k}\right) \leq M^{\prime \prime} k^{3} \log k, \quad \text { for } k \text { large enough. }
$$

Observe that the highest power (that is, $k^{3}$ ) comes from the second term in (4.2).

4.2. A trigonometric inequality. The proofs of the two remaining steps rest on an elementary inequality that we present in this subsection. As in 4, the key observation is

$$
|\sin \alpha| \geq\left|\sin 2^{n} \alpha\right| / 2^{n}, \quad n \in \mathbb{N}, \quad \alpha \in \mathbb{R} .
$$

Lemma 4.4. Let $r \geq 1$ and let $n_{0}>n_{1}>\cdots>n_{r} \geq 0$ be a finite decreasing sequence of natural numbers. If $2^{n_{j}} \varphi_{j}=\pi[2 \pi]$ (i.e. $2^{n_{j}} \varphi_{j}=\pi \bmod 2 \pi$ ), $j=$ $0, \ldots, r-1$, then

$$
\prod_{j=0}^{r-1}\left|\sin 2^{n_{j+1}-1}\left(\varphi-\varphi_{0}-\cdots-\varphi_{j}\right)\right| \geq\left(1 / 2^{n_{0}-n_{r}}\right)\left|\cos 2^{n_{0}-1} \varphi\right|, \quad \varphi \in \mathbb{R} .
$$

Proof. The proof is by induction. To treat the case $r=1$, we prove that

$$
\left|\sin 2^{n_{1}-1}\left(\varphi-\varphi_{0}\right)\right| \geq\left(1 / 2^{n_{0}-n_{1}}\right)\left|\cos 2^{n_{0}-1} \varphi\right| \text {. }
$$

Using (4.3) with $\alpha=2^{n_{1}-1}\left(\varphi-\varphi_{0}\right)$ and $n=n_{0}-n_{1}$ we obtain

$$
\left|\sin 2^{n_{1}-1}\left(\varphi-\varphi_{0}\right)\right| \geq\left(1 / 2^{n_{0}-n_{1}}\right)\left|\sin 2^{n_{0}-1}\left(\varphi-\varphi_{0}\right)\right| .
$$

But, since $2^{n_{0}} \varphi_{0}=\pi[2 \pi]$, $\left|\sin 2^{n_{0}-1}\left(\varphi-\varphi_{0}\right)\right|=\left|\cos 2^{n_{0}-1} \varphi\right|$ and the claim follows.

We now assume that the inequality is true for $r=k$ and prove it for $r=k+1$. The induction hypothesis applied to $\varphi-\varphi_{0}$ instead of $\varphi$ yields

$$
\prod_{j=1}^{k}\left|\sin 2^{n_{j+1}-1}\left(\left(\varphi-\varphi_{0}\right)-\varphi_{1}-\cdots-\varphi_{j}\right)\right| \geq\left(1 / 2^{n_{1}-n_{k+1}}\right)\left|\cos 2^{n_{1}-1}\left(\varphi-\varphi_{0}\right)\right|, \quad \varphi \in \mathbb{R} .
$$

Multiplying by the term corresponding to $j=0$, we obtain

$$
\begin{gathered}
\prod_{j=0}^{k}\left|\sin 2^{n_{j+1}-1}\left(\left(\varphi-\varphi_{0}\right)-\varphi_{1}-\cdots-\varphi_{j}\right)\right| \\
\geq \frac{1}{2^{n_{1}-n_{k+1}}}\left|\sin 2^{n_{1}-1}\left(\varphi-\varphi_{0}\right) \cos 2^{n_{1}-1}\left(\varphi-\varphi_{0}\right)\right| \\
=\frac{1}{2^{n_{1}-n_{k+1}+1}}\left|\sin 2^{n_{1}}\left(\varphi-\varphi_{0}\right)\right|, \quad \varphi \in \mathbb{R} .
\end{gathered}
$$

Another use of (4.3) with $n=n_{0}-n_{1}-1$ shows that

$$
\frac{1}{2^{n_{1}-n_{k+1}+1}}\left|\sin 2^{n_{1}}\left(\varphi-\varphi_{0}\right)\right| \geq \frac{1}{2^{n_{0}-n_{k+1}}}\left|\sin 2^{n_{0}-1}\left(\varphi-\varphi_{0}\right)\right|, \quad \varphi \in \mathbb{R} .
$$


The sine on the right-hand side is shown to be $\left|\cos 2^{n_{0}-1} \varphi\right|$ as in the case $r=1$.

4.3. Proof of Theorem 4.2. Let $X=X(E)$ and $B=X\left(2^{n}+1: k-1\right)$ with $2^{n}+1<k \leq 2^{n+1}$. We write

$$
k-1=2^{n}+2^{n_{1}}+\cdots+2^{n_{r}} \quad \text { with } n-1 \geq n_{1}>\cdots>n_{r} \geq 0,
$$

and, to simplify the notation,

$$
\begin{aligned}
n_{0} & =n+1, \\
d_{i} & =2^{n_{0}}+\cdots+2^{n_{i}}, \quad i=0, \ldots, r .
\end{aligned}
$$

Then, in view of Corollary 2.5, we have

$$
\begin{aligned}
B & =X\left(2^{n}+1: 2^{n}+\left(d_{1}-d_{0}\right)\right) \wedge \bigwedge_{i=1}^{r-1} X\left(2^{n}+\left(d_{i}-d_{0}\right)+1: 2^{n}+\left(d_{i+1}-d_{0}\right)\right) \\
& =\bigwedge_{i=0}^{r-1} \Re\left(E\left(d_{i}: d_{i+1}-1\right)\right) .
\end{aligned}
$$

Now using the structure properties of a Leja sequence (see Theorem 2.1] and (2.2)), we see that the points of $X\left(2^{n}+1, k-1\right)$ are certain modified Chebyshev points (see Subsection 3.5). Indeed, for $i \in\{0, \ldots, r-1\}$,

$$
E\left(d_{i}: d_{i+1}-1\right)=\rho_{i} \cdots \rho_{1} \rho_{0} E_{2^{n_{i+1}}}^{(i+1)}, \quad \text { with } \rho_{j}^{2^{n_{j}}}=-1,
$$

$$
\Re\left(E\left(d_{i}: d_{i+1}-1\right)\right)=\mathcal{T}_{2^{n_{i+1}}}^{\left(\beta_{0}+\cdots+\beta_{i}\right)}, \quad \text { with } \beta_{j}=\arg \rho_{j}=\left(2 t_{j}+1\right) \pi / 2^{n_{j}}, t_{j} \in \mathbb{Z} .
$$

This implies the following relation for the polynomial $w_{B}$ :

$$
w_{B}(x)=\prod_{i=0}^{r-1}\left\{T_{2^{n_{i+1}}}(x)-T_{2^{n_{i+1}}}\left(\cos \left(\beta_{0}+\cdots+\beta_{i}\right)\right)\right\} .
$$

It follows that for $a=\cos \varphi \in A=X\left(0: 2^{n}\right)$,

$$
\begin{aligned}
\max _{x \in[-1,1]} \frac{\left|w_{B}(x)\right|}{\left|w_{B}(a)\right|} & =\max _{t \in \mathbb{R}} \prod_{i=0}^{r-1} \frac{\left|\cos \left(2^{n_{i+1}} t\right)-\cos \left(2^{n_{i+1}}\left(\beta_{0}+\cdots+\beta_{i}\right)\right)\right|}{\left|\cos \left(2^{n_{i+1}} \varphi\right)-\cos \left(2^{n_{i+1}}\left(\beta_{0}+\cdots+\beta_{i}\right)\right)\right|} \\
& \leq 2^{r} \prod_{i=0}^{r-1} 1 /\left|\cos \left(2^{n_{i+1}} \varphi\right)-\cos \left(2^{n_{i+1}}\left(\beta_{0}+\cdots+\beta_{i}\right)\right)\right| .
\end{aligned}
$$

Now, a use of the sum-to-product formula for cosines, together with two applications of Lemma 4.4 (first with $\varphi_{i}=\beta_{i}$, then with $\varphi_{i}=-\beta_{i}$ ), enables us to bound the denominator in (4.13) and arrive at

$$
\max _{x \in[-1,1]} \frac{\left|w_{B}(x)\right|}{\left|w_{B}(a)\right|} \leq \frac{2^{2\left(n_{0}-n_{r}\right)}}{\cos ^{2}\left(2^{n_{0}-1} \varphi\right)} .
$$

It remains to recall that $n_{0}=n+1$ so that $2^{2\left(n_{0}-n_{r}\right)} \leq 2^{2 n+2}$ and observe that, since $A=\mathcal{L}_{2^{n}}, 2^{n_{0}-1} \varphi=2^{n} \varphi=0[\pi]$ so that $\cos ^{2}\left(2^{n_{0}-1} \varphi\right)=1$. This concludes the proof of Theorem 4.2 
4.4. Proof of Theorem 4.3. We still use the fact that, for $X=X(E)$ and $2^{n}+1<$ $k \leq 2^{n+1}$, we have $B=\bigwedge_{i=0}^{r-1} B_{i}$, where the underlying set of $B_{i}$ is $\mathcal{T}_{2^{n_{i+1}}}^{\left(\beta_{0}+\cdots+\beta_{i}\right)}$ with $\beta_{j}=\left(2 t_{j}+1\right) \pi / 2^{n_{j}}, t_{j} \in \mathbb{Z}$.

Using first Lemma 3.3 (with $N_{i}=B_{i}$ ) and then Lemma 3.5 to bound $\Delta\left(B_{j}\right)$, we obtain

$$
\Delta(B) \leq M \sum_{j=0}^{r-1} \max _{x \in[-1,1], a \in B_{j}} \frac{\left|w_{B \backslash B_{j}}(x)\right|}{\left|w_{B \backslash B_{j}}(a)\right|} \frac{\log \left(2^{n_{j+1}}+1\right)}{\left|\sin \left(2^{n_{j+1}}\left(\beta_{0}+\cdots+\beta_{j}\right)\right)\right|} .
$$

Now, just as in (4.13) (we just remove one factor), for $a=\cos \theta_{j} \in B_{j}$, we have

$$
\begin{aligned}
\max _{x \in[-1,1]} \frac{\left|w_{B \backslash B_{j}}(x)\right|}{\left|w_{B \backslash B_{j}}(a)\right|} & =\max _{t \in \mathbb{R}} \prod_{i=0, i \neq j}^{r-1} \frac{\left|\cos \left(2^{n_{i+1}} t\right)-\cos \left(2^{n_{i+1}}\left(\beta_{0}+\cdots+\beta_{i}\right)\right)\right|}{\left|\cos \left(2^{n_{i+1}} \theta_{j}\right)-\cos \left(2^{n_{i+1}}\left(\beta_{0}+\cdots+\beta_{i}\right)\right)\right|} \\
& \leq 2^{r-1} \prod_{i=0, i \neq j}^{r-1} 1 /\left|\cos \left(2^{n_{i+1}} \theta_{j}\right)-\cos \left(2^{n_{i+1}}\left(\beta_{0}+\cdots+\beta_{i}\right)\right)\right| .
\end{aligned}
$$

Again the sum-to-product formula for cosines transforms the right-hand side in a product of sines and it follows that the $j$-term in the right-hand side of (4.14) is bounded by the maximum when $a=\cos \theta_{j}$ runs over $B_{j}$ of

$$
\begin{aligned}
\log \left(2^{n_{j+1}}+1\right) \prod_{i=0, i \neq j}^{r-1}\left|\sin ^{-1}\left(2^{n_{i+1}-1}\left(\theta_{j}-\beta_{0}-\cdots-\beta_{i}\right)\right)\right| \\
\times \prod_{i=0}^{r-1}\left|\sin ^{-1}\left(2^{n_{i+1}-1}\left(\theta_{j}+\beta_{0}+\cdots+\beta_{i}\right)\right)\right| .
\end{aligned}
$$

Here we used the fact that

$$
\left|\sin \left(2^{n_{j+1}-1}\left(\theta_{j}+\beta_{0}+\cdots+\beta_{j}\right)\right)\right|=\left|\sin \left(2^{n_{j+1}}\left(\beta_{0}+\cdots+\beta_{j}\right)\right)\right|,
$$

which enabled us to insert the isolated sine in (4.14) into the second product of (4.17). To prove (4.18), we observe that, since $a=\cos \theta_{j} \in B_{j}$, we have

$$
2^{n_{j+1}} \theta_{j}=2^{n_{j+1}}\left(\beta_{0}+\cdots+\beta_{j}\right)[2 \pi] .
$$

We now estimate independently both products in (4.17). The same bound is valid for every $a \in B_{j}$ and therefore provides an upper bound for the maximum over $B_{j}$ as required.

I) We start with the first product. In view of (4.19), since $n_{i+1}>n_{j+1}$ whenever $i<j$, we have

$$
2^{n_{i+1}}\left(\theta_{j}-\beta_{0}-\cdots-\beta_{i}\right)=2^{n_{i+1}}\left(\beta_{i+1}+\cdots+\beta_{j}\right)[2 \pi], \quad 0 \leq i<j .
$$

On the other hand, since $2^{n_{s}} \beta_{s}=\pi[2 \pi]$, we also have

$$
2^{n_{i+1}}\left(\beta_{i+1}+\cdots+\beta_{j}\right)=\pi[2 \pi], \quad 0 \leq i<j .
$$

Thus the absolute value of the first $j$ sines equals 1 , and we just need to estimate

$$
\prod_{i=j+1}^{r-1}\left|\sin 2^{n_{i+1}-1}\left(\theta_{j}-\beta_{0}-\cdots-\beta_{i}\right)\right| \text {. }
$$


To do that, we apply Lemma 4.4 with $\varphi=\theta_{j}-\beta_{0}-\cdots-\beta_{j}$. We obtain the lower bound $\left(1 / 2^{n_{j+1}-n_{r}}\right)\left|\cos 2^{n_{j+1}-1}\left(\theta_{j}-\beta_{0}-\cdots-\beta_{j}\right)\right|$. Yet in view of (4.19) this cosine equals \pm 1 , and we obtain

$$
\prod_{i=0, i \neq j}^{r-1}\left|\sin ^{-1}\left(2^{n_{i+1}-1}\left(\theta_{j}-\beta_{0}-\cdots-\beta_{i}\right)\right)\right| \leq 2^{n_{j+1}-n_{r}} .
$$

Note that in the case $j=r-1$, the whole product equals 1 , which obviously implies the inequality. The inequality is likewise satisfied in the case $r=1$ (for which the product is empty).

II) We now turn to the second product in (4.17). We obtain an upper bound again using Lemma 4.4 as in (4.6). Indeed, we have

$$
\begin{aligned}
& \prod_{i=0}^{r-1}\left|\sin \left(2^{n_{i+1}-1}\left(\theta_{j}+\beta_{0}+\cdots+\beta_{i}\right)\right)\right| \\
& \quad=\left|\sin \left(2^{n_{1}-1}\left(\theta_{j}+\beta_{0}\right)\right)\right| \prod_{i=1}^{r-1}\left|\sin \left(2^{n_{i+1}-1}\left(\theta_{j}+\beta_{0}+\cdots+\beta_{i}\right)\right)\right| .
\end{aligned}
$$

Applying Lemma 4.4 with $\varphi=\theta_{j}+\beta_{0}$ to the second factor on the left-hand side, we obtain

$$
\prod_{i=1}^{r-1}\left|\sin \left(2^{n_{i+1}-1}\left(\theta_{j}+\beta_{0}+\cdots+\beta_{i}\right)\right)\right| \geq \frac{1}{2^{n_{1}-n_{r}}}\left|\cos \left(2^{n_{1}-1}\left(\theta_{j}+\beta_{0}\right)\right)\right| .
$$

It follows that

$$
\begin{aligned}
& \prod_{i=0}^{r-1}\left|\sin \left(2^{n_{i+1}-1}\left(\theta_{j}+\beta_{0}+\cdots+\beta_{i}\right)\right)\right| \\
& \geq \frac{1}{2^{n_{1}-n_{r}+1}} \mid 2 \sin \left(2^{n_{1}-1}\left(\theta_{j}+\beta_{0}\right)\right) \\
& \quad=\frac{1}{2^{n_{1}-n_{r}+1}}\left|\sin \left(2^{n_{1}}\left(\theta_{j}+\beta_{0}\right)\right)\right| .
\end{aligned}
$$

Thus we get the following upper bound for the second product in (4.17),

$$
2^{n_{1}-n_{r}+1} /\left|\sin 2^{n_{1}}\left(\theta_{j}+\beta_{0}\right)\right| \text {. }
$$

However, since for every $s, \beta_{s}=\left(2 t_{s}+1\right) \pi / 2^{n_{s}}$ and $\theta_{j}=\beta_{0}+\cdots+\beta_{j}+2 q_{j} \pi / 2^{n_{j+1}}$ with $t_{s}, q_{j} \in \mathbb{Z}$, we have

$$
\begin{aligned}
2^{n_{1}}\left(\theta_{j}+\beta_{0}\right)=2^{n_{1}}\left(2 \beta_{0}+\beta_{1}\right. & \left.+\cdots+\beta_{j}+2 q_{j} \pi / 2^{n_{j+1}}\right) \\
& =2^{n_{1}+1} \beta_{0}+p \pi=\frac{\left(2 t_{0}+1\right) \pi}{2^{n_{0}-n_{1}-1}}+p \pi \quad \text { with } p \in \mathbb{Z} .
\end{aligned}
$$

Note that since $n_{0}=n+1$ and $n>n_{1}$ we have $n_{0}-n_{1}-1>0$. This shows that $\left|\sin 2^{n_{1}}\left(\theta_{j}+\beta_{0}\right)\right| \geq \sin \left(\pi / 2^{n_{0}-n_{1}-1}\right) \geq 2 / 2^{n_{0}-n_{1}-1}=1 / 2^{n_{0}-n_{1}-2}$. We have therefore proved

$$
\prod_{i=0}^{r-1}\left|\sin ^{-1}\left(2^{n_{i+1}-1}\left(\theta_{j}+\beta_{0}+\cdots+\beta_{i}\right)\right)\right| \leq 2^{n_{1}-n_{r}+1} \cdot 2^{n_{0}-n_{1}-2}=2^{n_{0}-n_{r}-1} \leq 2^{n} .
$$


III) It remains to insert (4.21) and (4.22) in (4.14) with the aid of (4.17). Indeed, we obtain

$\Delta(B) \leq M \sum_{j=0}^{r-1} 2^{n_{j+1}-n_{r}} \cdot 2^{n} \cdot \log \left(2^{n_{j+1}}+1\right)$

$$
\leq M 2^{n} \sum_{j=0}^{r-1} 2^{n_{j+1}} \log \left(2^{n_{j+1}}+1\right) \leq M 2^{n} \log \left(2^{n}+1\right) \sum_{j=0}^{r-1} 2^{n_{j+1}}=O\left(2^{2 n} \log 2^{n}\right) .
$$

This concludes the proof of Theorem 4.3 .

\section{ACKNOWLEDGEMENT}

The work of the second author is supported by a $\mathrm{PhD}$ fellowship from the Vietnamese government.

\section{REFERENCES}

[1] L. Białas-Cież and J.-P. Calvi. Pseudo Leja sequences. Ann. Mat. Pura e Appl., 191:53-75, 2012.

[2] L. Brutman. Lebesgue functions for polynomial interpolation-a survey. Ann. Numer. Math., 4(1-4):111-127, 1997. The heritage of P. L. Chebyshev: a Festschrift in honor of the 70th birthday of T. J. Rivlin. MR 1422674 (97m:41003)

[3] J.-P. Calvi. Intertwining unisolvent arrays for multivariate Lagrange interpolation. Adv. Comput. Math., 23(4):393-414, 2005. MR2137463(2006a:41002)

[4] J.-P. Calvi and Phung Van Manh. On the Lebesgue constant of Leja sequences for the unit disk and its applications to multivariate interpolation. J. Approx. Theory, 163(5):608-622, 2011. MR 2784514

[5] H. Ehlich and K. Zeller. Auswertung der Normen von Interpolationsoperatoren. Math. Ann., 164:105-112, 1966. MR0194799 (33:3005)

[6] A. P. Goncharov. On growth of norms of Newton interpolating operators. Acta Math. Hungar., 125(4):299-326, 2009. MR2564431(2011a:41030)

[7] T. H. Gronwall. A sequence of polynomials connected with the $n$th roots of unity. Bull. Amer. Math. Soc., 27:275-279, 1921. MR1560416

[8] T. J. Rivlin. An introduction to the approximation of functions. Dover Publications Inc., New York, 1981. Corrected reprint of the 1969 original, Dover Books on Advanced Mathematics. MR634509 (83b:41001)

[9] R. Taylor and V. Totik. Lebesgue constants for Leja points. IMA J. Numer. Anal., 30:462-486, 2010. MR2608468 (2011d:41003)

Institut de Mathématiques de Toulouse, Université Paul Sabatier, Toulouse, France E-mail address: jean-paul.calvi@math.univ-toulouse.fr

Institut de Mathématiques, Université De Toulouse III And CNRS (UMR 5219), 31062, Toulouse Cedex 9, France - and - Department of Mathematics, Hanoi University of Education, 136 Xuan Thuy street, Caugiay, Hanoi, Vietnam

E-mail address: manhlth@gmail.com 